\title{
A Procedure for Dex-induced Gene Transactivation in Arabidopsis Ovules
}

\author{
Jasmin Schubert \\ University of Zürich \\ Yanru Li \\ University of Zürich \\ Marta A. Mendes \\ University of Milan \\ Hugh Dickinson \\ University of Oxford \\ Célia Baroux ( $\nabla$ cbaroux@botinst.uzh.ch ) \\ University of Zürich https://orcid.org/0000-0001-6307-2229
}

\section{Methodology}

Keywords: pOP/LhGR, Dex induction, ovule development, Arabidopsis

Posted Date: June 29th, 2021

DOl: https://doi.org/10.21203/rs.3.rs-652621/v1

License: (c) (1) This work is licensed under a Creative Commons Attribution 4.0 International License. Read Full License

Version of Record: A version of this preprint was published at Plant Methods on March 29th, 2022. See the published version at https://doi.org/10.1186/s13007-022-00879-x. 


\section{Abstract}

Background: Elucidating the genetic and molecular control of plant reproduction requires the deployment of functional approaches based, for instance, on reverse or forward genetic screens. The loss-of-function of essential genes, however, may lead to plant lethality prior to reproductive developmentt or to the formation of sterile structures before the organ-of-interest can be analysed. In these cases, inducible approaches that enable a spatial and temporal control of the genetic perturbation are extremely valuable. Genetic induction in reproductive organs, such as the ovule, deeply embedded in the flower, is a delicate procedure that requires both optimization and validation.

Results: Here we report on a streamlined procedure enabling reliable induction of gene expression in Arabidopsis ovule and anther tissues using the popular pOP/LhGR Dex-inducible system. We demonstrate its efficiency and reliability using fluorescent reporter proteins and histochemical detection of the GUS reporter gene.

Conclusion: The pOP/LhGR system allows for a rapid, efficient and reliable induction of transgenes in developing ovules without compromising developmental progression. This approach opens new possibilities for the functional analysis of candidate regulators in sporogenesis and gametogenesis, which are otherwise affected by early lethality of conventional, stable mutants.

\section{Background}

To elucidate the genetic and molecular mechanisms of a developmental process, a conventional approach consists of analyzing mutant lines isolated in a forward- or reverse genetic screen. However, the stable expression of a gain- or a loss-of-function mutation in a gene acting at several stages of plant development can be deleterious prior to the formation of a tissue/organ of interest, thus impairing on the analysis. This is a difficulty particularly faced when analysing the role of essential genes with pleiotropic effects during reproduction. Their disruption following chemically-induced mutagenesis, insertional mutagenesis or gene editing may abort development or organ growth before reproductive development. In such cases, inducible transactivation systems offer an elegant solution for controlling a genetic perturbation in a conditional manner, be it an artificial miRNA for downregulation or an engineered mutant variant of the protein of interest. Combining a tissue-specific promoter driving the activating factor with the selective application of the inducer, such systems can provide exquisite temporal and spatial control. However, inducible systems often require that, both the expression control and application procedure have to be adapted to the organ/tissue of interest and validated for functional analyses.

Different chemically-inducible system exist that rely on diverse compounds such as ethanol (1) or animal hormones, including estradiol (2) and dexamethasone (3). Among them, the POP/LhGR dexamethasone (Dex)-inducible system has been very popular and widely used. It relies on the Dex-induced translocation of the synthetic transcription factor LhGR into the nucleus. Prior to induction, LhGR is retained in the cytoplasm by the HSP90 chaperone but once in the nucleus, it binds to the pOP6 promoter at optimally- 
spaced repeats in (3). The pOP/LhGR-inducible system had been demonstrated to induce tight and reliable gene expression control and has been widely used in Arabidopsis, Cardamine, tobacco and crop plants including tomato, maize and rice (4-11) and references therein. Reports of the successful use of the system include the induction of transcription factors, toxins for cell ablations, the cre/lox system for recombination, RNAi for gene downregulation and other cellular components, in leaves, roots, cambium tissue, zygote, embryo and apical meristems (see for instance $(9,10,12-18)$ inter alia. Resources have also been recently established of driver/responder lines for expression in different cell types in Arabidopsis and other species $(8,9)$. Dex can be applied by watering whole plants, by supplementing the growth medium of cell cultures, seedlings or tissue segments in vitro (9). However, localised induction in tissues that are embedded in floral organs poses technical difficulties due to their relative inaccessibility. Here we report on an application procedure that has been optimized for inducing transgene expression in developing ovules of Arabidopsis. This strategy largely preserves viability and ovule developmental progression. The solvent used for the Dex solution however induces a mild reduction in fertility, requiring the use of stringent mock controls. This application method allows for a durable (over several days) and efficient (throughout cell layers) transgene expression in developing ovules, and is also applicable to anthers.

\section{Methods}

\section{Plant material and growth conditions}

Arabidopsis seeds were sterilized (10 min in $0.03 \%$ bleach, $0.05 \%$ Triton X-100), washed three times in water and briefly incubated in $70 \% \mathrm{EtOH}$ before sowing on germination medium (0.5xMurashige and Skoog (MS) salts (CAROLINE 19-5700, USA), 1\% (w/v) Bactoagar pH 5.6) or storing dry on filter paper $\left(4^{\circ} \mathrm{C}\right)$ until sown. Seeds were stratified 2 to 4 days at $4^{\circ} \mathrm{C}$ before transfer to growth incubators (Percival) with long day conditions ( 16 hours light $[120 \mu \mathrm{E} \mathrm{m}-2 \mathrm{~s}-1]$ at $21^{\circ} \mathrm{C}$ and 8 hours dark at $16^{\circ} \mathrm{C}$ ). 2 weeks after germination, seedlings were transferred to soil and grown under long day conditions ( $16 \mathrm{~h} \mathrm{light/} 8 \mathrm{~h}$ dark; $22^{\circ} \mathrm{C} / 18^{\circ} \mathrm{C}$, respectively).

The n/s3xmVenus construct (Joop Vermeer, University of Zurich) was subcloned in a Gateway LR reaction into the pRPS5a-pop6-LhGR2 vector (9). The construct was introduced into Agrobacterium tumefaciens (GV3101) and transformed to Arabidopsis Col-0 plants. Primary transformants were selected based on BASTA resistance, and scored as positive based on GUS reporter assays and $n / s 3 x m$ Venus signals $16 \mathrm{~h}$ following a $10 \mu \mathrm{M}$ Dex-treatment.

The H1.1-RFP ( $p$ RPS5a > > H1.1-RFP) construct was made by assembling a synthetic H1.1-tagRFP-T coding sequence (H1.1: AT1G06760, tagRFP-T: GenBank: ACD03281 (28), synthesis: GeneART, Invitrogen) into the pRPS5a-pop6-LhGR vector (9). The constructs were transformed via Agrobacterium tumefaciens (GV3101) to Col-0 plants. Positive T1s were identified based on BASTA resistance selection, GUS reporter assay and RFP signals $16 \mathrm{~h}$ after $10 \mu \mathrm{M}$ Dex-treatment. 
The $p R P S 5 a>>\triangle K$ Kinesin-YFP construct was made by amplifying a $957 \mathrm{bp}$ fragment encompassing the coiled-coil domains of At3g20150 (recently termed Kinesin-12F (29) from Arabidopsis Col-0 cDNA using primers 5'-TTTGGCGCGCCCACCATGGGAGCAAGTAATGGAGA-3' and 5'-

AAAGGCGCGCCTTGCTCCCTACATACCTTCTTCCTC-3'. Following Ascl digestion of the PCR product, the fragment was inserted into pENTRY-YFP (30). This was then recombined with pOpIN2 (9) through an LR reaction to generate the vector $p R P S 5 a>>\triangle$ Kinesin-YFP (alternative name: pOpIN2/RPS5a > Dex > KIN12Fcoiled-coil).

For $p S P L>>$ amiMET1 and $p S P L>>$ amiFIE the putative promoter regions plus the $5^{\prime} \mathrm{UTR}$ and the $3^{\prime} \mathrm{UTR}$ of the SPL locus (AT4G27330) were amplified as described $(31,32)$. The fragments were cloned into pBIN-LR-LhGR2 using Gateway-compatible sites as described (9) to generate the $p S P L:: L h G R$ vector and an Ascl $p S P L .: L h G R$ fragment was cloned into pOPIn2 (9) to generate $p S P L .: L h G R / p O P I n 2$. The amiRNAs against FIE (AT3G20740) and MET1 (AT5G49160) were amplified using genomic DNA Col_0 as template as described (33). The amiRNA target sequences were TCGTCCAATTCCCTGTATTA and TAGCCAACAGAGTATTACTGC for FIE and MET1, respectively. The amiRNA were cloned into a PUC Entry clone and subcloned by LR reaction (Invitrogen) into the $p S P L:: L h G R / p O P I n 2$ vector.

\section{Preparation of Dex- stock and induction solution}

The stock solution of $10 \mathrm{mM}$ Dex in either pure DMSO or $100 \% \mathrm{EtOH}$ is stored at $-20^{\circ} \mathrm{C}$ and can be kept for up to one year. The final induction solution consists of $10 \mu \mathrm{M}$ Dex (SIGMA-ALDRICH, D4902) (prepared from the stock solution by diluting with water) and 0.01\% Silwet L-77 (Lehle Seeds, USA, Cat. VIS-01). Importantly, the induction solution should be prepared freshly and stored at $4^{\circ} \mathrm{C}$ for a maximum of three days.

\section{$\beta$-Glucuronidase reporter assay}

Carpels are gently opened under the dissecting scope, immerged in GUS staining solution (Triton X-100 10\%, EDTA 10 mM, Ferrocyanide 2 mM, Ferricyanide 2 mM, Na2HP04 100 mM, NaH2P04 100 mM, $\beta$ Glucuronidase $2 \mathrm{mM}$.) and vacuum infiltrated for $5 \mathrm{~min}$. The samples are then incubated for $2 \mathrm{hrs}$ at $37^{\circ} \mathrm{C}$, briefly rinsed with $50 \mathrm{mM}$ phosphate buffer ( $\mathrm{pH}$ 6.8) (0.2 M NaH2PO4, $2 \mathrm{M} \mathrm{Na2HPO3)}$ and mounted freshly in 80 \% glycerol for microscopy imaging (DIC settings, Leica DMR, Leica microsystems GmbH, Germany).

\section{Fluorescence imaging and image processing}

Carpels were collected from treated flowers and placed on a clean microscope slide. Ovule primordia were gently exposed using dissecting needs in the mounting solution consisting in $0.5 \mathrm{xMS}$ or renaissance staining solution (4\% paraformaldehyde; 1:2000 renaissance; $10 \%$ glycerol; $0.05 \%$ DMSO in 1x PBS (modified from (34)). Images were collected using a confocal laser scanning microscope using a 63XGLY APO NA1.4 objective (SP5R, Leica microsystems, Germany). Images were processed (partial or full projections, 3D rendering and orthogonal sections, as indicated in the figure legend) using Imaris (Bitplane, Switzerland). 


\section{RT-PCR analysis}

Quantitative real-time RT-PCR experiments were performed using cDNA obtained from inflorescences. Total RNA was extracted using the Qiagen RNA extraction Kit. Ambion TURBO DNA-free DNase kit was used to eliminate genomic DNA contamination according to the manufacturer's instructions (http://www.ambion.com/). The ImProm-IITM reverse transcription system (Promega) was used to retrotranscribe the treated RNA. Transcripts were detected using a Sybr Green Assay (iQ SYBR Green Supermix; Bio-Rad) using UBIQUITIN10 (AT4G05320) as a reference gene. Assays were done in triplicate using a Bio-Rad iCycler iQ Optical System (software v.3.0a). Forward and reverse primers for FIE: TCTGAACACCTGCCTCACAG and TGTGACTGAGAACCGCTGTC, respectively; for MET1:

GTGTGGCGTTAATGGGAAC and TCTCCATGACCCACAAGACTC, respectively. Primer specificity tests and qPCR experiments were performed as described (35) with the following cycling conditions: $3 \mathrm{~min}$ at $95^{\circ} \mathrm{C}$ followed by 45 cycles of $10 \mathrm{~s}$ at $95^{\circ} \mathrm{C}, 1 \mathrm{~s}$ at $55^{\circ} \mathrm{C}, 30 \mathrm{~s}$ at $72^{\circ} \mathrm{C}$, and $15 \mathrm{~s}$ at the optimal acquisition temperature. FIE and MET1 transcript levels were normalized with UBQ10 levels.

\section{Scoring of ovules stages}

For experiments shown in Fig. 4A, floral buds treated by Dex, Mock or water as described above were fixed in Acetic Acid:EtOH 3:1, stored overnight at $4^{\circ} \mathrm{C}$, transferred in $70 \% \mathrm{EtOH}$, mounted in clearing solution (chloral hydrate: water: glycerol 8:2:1 v:v:w) and imaged under wide-field light microscopy with Nomarski optical settings (DMR, Leica microsystems, Germany). Ovule stages were scored according to the nomenclature for ovule primordia $(20,36)$ and ovules $(37)$

\section{Fertility analysis}

Single inflorescences were induced twice (at 0 and 16hrs) with $10 \mu \mathrm{M}$ Dex, $0.01 \%$ Silvwet or Mock solutions including the equivalent share of $\mathrm{EtOH}$ or DMSO as indicated on the graph. After two weeks the first 10 siliques of the induced inflorescences were collected for seed set analysis.

\section{Results}

\section{Dexamethasone application on inflorescences}

Ovule primordia are the site of the somatic-to-reproductive transition where in the female meiocyte differentiates (19). In Arabidopsis, ovule primordia are small, digit-shape structures of 10 to $50 \mu \mathrm{M}$ length depending on the developmental stage, encapsulated in the carpel itself, and enclosed in a flower bud (20). Flower buds containing developing primordia are ca. $1-2 \mathrm{~mm}$ large and tightly packed in the apical inflorescence. Due to the relative inaccessibility of ovule primordia, chemical induction of gene expression is thus not trivial. Notably, the inducer has to be applied locally on buds which can be brittle, and handling should ideally avoid wounding while enabling efficient penetration through the floral corolla (sepals, petals) and the carpel wall. 
We found that local application using a small soft-hair painting brush was not disruptive and allowed a precise application. Diluting Dex in a physiological medium such as half-MS was not sufficient to induce primordia inside the carpel. We found, however, that adding a surfactant such as Silwet ${ }^{\circ} L-77$ already used in Agrobacterium infiltration (Clough and Bent 1998) enabled a good induction. Yet, prolonged exposure to this surfactant led to tissue damage and developmental arrest of the bud. Details of the procedure are as follows: petals and sepals of individual flower buds at the appropriate stage are gently pulled apart under a stereomicroscope using a dissecting needle, without damaging the tissues, (Fig. 1a). Then, a drop of freshly prepared $10 \mu \mathrm{M}$ Dex solution (or mock solution for control) is applied on the bud with a soft-hair paint brush. The application is monitored under the stereomicroscope and care is taken that the inflorescence remains wet but not engulfed in a large drop of induction medium (which would lead to tissue damage overnight). After application, plants should be covered overnight (Fig. 1b), keeping them uncovered reduced the reliability of induction. Keeping a moist environment may prevent drying of the inducing medium before it diffuses to underlying layers. The buds are harvested after $\sim 16 \mathrm{~h}$ incubation and prepared for direct microscopy observation or for histological staining (Fig. 1c).

We illustrate the efficiency of this induction protocol Fig. $1 \mathrm{~d}$ and following figures. Figure $1 \mathrm{~d}$ shows the induction of $n / s 3 x$-Venus and GUS reporter genes under the control of the RPS5a promoter (9). In this case both reporter genes are on the same construct and simultaneously activated thanks to the bidirectional activity of the pOp promoter (3).

Important factors influencing the success of the induction solution are: (i) the age of the Dex solution; it should be prepared freshly and stored at $4^{\circ} \mathrm{C}$ for a maximum of three days. $10 \mathrm{mM}$ Dex stock solutions either in DMSO or EtOH can be stored at $-20^{\circ} \mathrm{C}$. (ii) health and age status of the plant. well-watered, healthy (uninfected/free of pests) young plants are better responding; the primary inflorescence should be used soon after emergence from the rosette. (iii) repeated induction: weak induction (due to handling, older plants, weakly expressing reporter) can be addressed by a repeated treatment $12-16 \mathrm{hrs}$ after the first, while minimising stress-inducing tissue damage .

\section{Efficient induction in carpels and anthers of various reporter genes}

Under our conditions different reporters targeting distinct cellular compartments could be induced in the ovule primordium. Seventeen hours following the application of the inducer ( $17 \mathrm{hpi}$, hours post induction) we were able to detect robust expression of fluorescent proteins such as YFP and RFP, targeted in the ER (Fig. 2a), nucleoplasm (Fig. 2b), chromatin (Fig. 2c), or the GUS reporter enzyme in the cytoplasm (Fig. 2d). To further validate these conditions functionally, we induced transgenes expressing amiRNA constructs targeting FERTILIZATION INDEPENDENT ENDOSPERM1 (FIE) and METHYLTRANSFERASE1 (MET1) and confirmed a reduction in transcript levels in young flower buds $48 \mathrm{~h}$ post Dex induction (Fig. 2e).

For meaningful use in gene expression control in reproductive organs the chemical inducer must reach all tissue and cell types throughout the organ. For this reason we investigated the efficiency of the 
application protocol described in Fig. 1, confirming that induction was efficient throughout the carpel (Fig. 3a,b), throughout the cell layers of ovule primordia (Fig. 3c), and was effective at different stages (Fig. 3d). Importantly, the tissues induced in our tests display expected cell-type specific expression (Fig. 3e) while being tightly regulated (Fig. 3h) as formerly described $(3,9)$.

\section{Effect of chemical induction on ovule development and plant fertility}

One question arising from this application procedure is whether the mechanical and chemical treatment itself affects reproductive development. We therefore monitored three aspects: (i) developmental progression of ovules in the carpel, (ii) Spore mother cell (SMC) differentiation in the ovule as a marker of functionality, and (iii) fertility. For (i) we fixed and cleared carpels 5 and 9 days post inductive treatment (dpi) using a Dex, Mock or water solution and scored the developmental stages. Our rationale was that if the treatment had a negative impact on development, we should observe a larger fraction of younger ovules, i.e. delayed in their developmental progression, in the Dex-treated samples compared to the Mock or water samples. We did not find evidence for such a developmental delay at $5 \mathrm{dpi}$ and $9 \mathrm{dpi}$, corresponding to ovules at the sporogenesis and gametogenesis stages, respectively (Fig. 4a, Additional File 1). Furthermore, and to address the second aspect (ii), we verified that treated ovules recapitulated a regular development, notably by monitoring one marker of the somatic-to-reproductive transition, scoring $\mathrm{H} 1.1$ eviction in the SMC of ovules expressing a Dex inducible H1.1-RFP variant ( $p R P S 5 a>>$ H1.1-RFP line) and comparing it to a non-inducible, control line expressing H1.1-GFP (21). 16hpi, H1.1-RFP was expressed throughout the primordium while at $5 \mathrm{dpi}$, a majority of ovules showed $\mathrm{H} 1.1$ eviction in the SMC in both the Dex-inducible and control lines (Fig. 4b, Additional File 1). Finally, we analyzed fertility (iii) by scoring the proportion of normal seeds (vs aborted seeds or unfertilized ovules) in plants treated with a Dex, a Mock or a Water solutions, and also by comparing different solvents for Dex $(0.25 \%$ DMSO, $0.4 \%$ $\mathrm{EtOH}, 1 \% \mathrm{EtOH}$, Fig. 4c, Additional File 1). While we discovered the the treatment itself to induce a variable seed set, with some siliques showing a high number of young aborted seeds, Dex in DMSO induced a moderate, but significant, reduction in seed set $(67.4 \% \pm 2.0, n=3474$ seeds, 71 siliques) compared to Mock DMSO-treated plants ( $82.7 \% \pm 2.8, n=2041$ seeds, 40 siliques). A similar effect was found for Dex treated plants when using Ethanol as a solvent, but only in one out of two replicates (using different EtOH concentrations). Considering the abortion rate in the water-treated sample, this may reflect mechanical handling rather than chemical treatment itself.

Collectively, this analysis indicates that Dex treatment does not significantly alter ovule primordium developmental progression but that it impacts on fertility, although to a limited extent.

\section{Discussion}

Chemically inducible systems offer the ability to temporally control transgene expression. In combination with tissue-specific promoters driving the activator construct, such systems ultimately allow for fine tuning gene activation at a spatial level. However, application of the inducer remains problematic when 
the cells-of interest are deeply embedded in complex tissues, such as meiocytes and gametes. In addition, induction by watering is not always an option if transgene activation is expected to be deleterious to plant development ahead of the stage of study. To circumvent this, local application of the inducer is thus preferable. Here, we designed and tested a protocol enabling robust, rapid and efficient induction throughout the carpels and anthers of an inflorescence. The application formula contains the widely used non-toxic detergent Silwet L-77 (22) at a $0.05 \%$ concentration which proved essential for a good penetration of the inducer in the floral tissue. We found that one application of $10 \mu \mathrm{M}$ Dex with a soft-hair paintbrush to whole inflorescences is enough to allow a robust induction of transgene expression in all ovules of the carpel. We also observed that if plants are not covered after induction, the treated tissue dries quickly and induction can fail. Covering the treated plant thus appeared as a critical step. Despite the robustness and usefulness of the application protocol, Dex-application using even a low concentration of solvent slightly affects the plant's fertility. Under our conditions, treating young buds containing ovule primordia, reduced seed set was principally a result of ovule infertility, but not seed abortion. For this reason, careful comparison with mock-treated plants should be made. However, in our studies, Dex application failed to affect developmental progression of ovule primordia, underlining the subtle effect of the Dex on fertility.

Compared to other chemical induction using ethanol (1) or estradiol (2) the pOP/LhGR system has several advantages, including a tight control of gene transactivation, versatility of application among plant species and tissue/cell-types (see $(8,9)$ and references in the introduction). But, so far, not many studies used the pOP/LhGR system directly on reproductive organs. Dex-mediated induction either of directly GR-tagged proteins or via the pOP/LhGR system has been reported for studying developmental or cellular processes in Arabidopsis ovules and embryo $(12,23)$. Here Dex-treatment was usually applied in a systematical manner via watering or sprayed on the whole plant. This is a good solution when the promoter used in the driver construct is temporally and spatially very specific. However, for developmental studies covering ovule development, starting from primordium emergence until the mature femalegametophyte bearing ovule, it is challenging to find stage-specific promoters. For instance, the KNUCKLE gene promoter used to drive a YFP reporter specifically in the female SMC (24) is in fact also active during shoot apical meristem development (25). This is also true for many other promoters formally used in reporter constructs for ovule development analyses. In such cases, whole-plant induction may well create adverse effects ahead of the stage of interest at which the transgene is targeted, potentially interfering with a cellular process to be studied during ovule development. Hence, the application procedure as reported here enables the employment of constitutive or tissue-specific promoters, yet induces transactivation only during a specific developmental window. This extends the spectrum of application of the pOP/LhGR Dex-inducible system $(3,8,9)$ to young Arabidopsis ovules.

\section{Conclusion}

Arabidopsis ovules are difficult to study microscopically due to their relative inaccessibility in the carpel, and to perturb genetically due to a lack of specific promoters enabling cell- and stage-specific perturbations. The pOP/LhGR, Dex-induction system offers a good solution to induce transgenes 
expression locally and at a specific stage. We present here an application protocol, with only a moderate and controllable effect on fertility due to manipulation, that is demonstrated to provide efficient and reliable time-course analysis of tagged protein expression following induction and induced gene downregulation in the Arabidopsis ovule. In combination with 3D cell-based analyses of ovule patterning $(26,27)$, inducible gene transactivation as described here completes the experimental toolkit for ovule developmental analysis.

\section{Declarations}

\section{Ethics approval and consent to participate}

Not applicable

\section{Consent for publication}

Not applicable

\section{Availability of data and materials}

The reporter lines used in the study are available from the corresponding author upon request.

\section{Competing interests}

The authors declare that they have no competing interests.

\section{Funding}

This work was supported by a grant from the National Swiss Science foundation to CB (SNSF 31003A_179244 and 310030_185186), the University of Zürich and a grant from Linea 2 Università degli studi di Milano PSR2015-1718LCOLO_12 to MM.

\section{Authors' contributions}

JS generated the H1.1-RFP and n/sVenus inducible lines, JS and YL performed the induction, imaging and quantification, confocal images were rendered and processed by JS and CB, MM generated the SPL >>amiRNA lines and performed the related induction, GUS staining and qRT-PCR experiments. IM generated the $\mathrm{POP} /$ LhGR vectors used in this study and provided the unpublished $p R P S 5 a>>\triangle k i n e s i n-Y F P$ line. CB prepared the figures, JS and CB wrote the manuscript. The work has received support from the European Union's Horizon 2020 research and innovation programme under the Marie Skłodowska-Curie grant agreement No 872417, Project MAD.

\section{Acknowledgements}

We thank Joop Vermeer (University of Neuchatel, Switzerland) for sharing the n/sVenus construct, Monika Kalde (Oxford University, UK) for sharing the pRPS5a >> 
discussions and providing access to a high-end research infrastructure and TA support.

\section{Competing interests}

The authors declare no competing interests.

\section{References}

1. Roslan HA, Salter MG, Wood CD, White MR, Croft KP, Robson F, et al. Characterization of the ethanolinducible alc gene-expression system in Arabidopsis thaliana. Plant J. 2001;28(2):225-35.

2. Zuo J, Niu QW, Chua NH. Technical advance: An estrogen receptor-based transactivator XVE mediates highly inducible gene expression in transgenic plants. Plant J. 2000;24(2):265-73.

3. Craft J, Samalova M, Baroux C, Townley H, Martinez A, Jepson I, et al. New pOp/LhG4 vectors for stringent glucocorticoid-dependent transgene expression in Arabidopsis. Plant J. 2005;41(6):899918.

4. Vlad D, Abu-Jamous $B$, Wang P, Langdale JA. A modular steroid-inducible gene expression system for use in rice. BMC Plant Biol. 2019;19(1):426.

5. Li XR, Deb J, Kumar SV, Ostergaard L. Temperature Modulates Tissue-Specification Program to Control Fruit Dehiscence in Brassicaceae. Mol Plant. 2018;11(4):598-606.

6. Samalova M, Brzobohaty B, Moore I. pOp6/LhGR: a stringently regulated and highly responsive dexamethasone-inducible gene expression system for tobacco. Plant J. 2005;41(6):919-35.

7. Hajheidari M, Wang Y, Bhatia N, Vuolo F, Franco-Zorrilla JM, Karady M, et al. Autoregulation of RCO by Low-Affinity Binding Modulates Cytokinin Action and Shapes Leaf Diversity. Curr Biol. 2019;29(24):4183-92. e6.

8. Schurholz AK, Lopez-Salmeron V, Li Z, Forner J, Wenzl C, Gaillochet C, et al. A Comprehensive Toolkit for Inducible, Cell Type-Specific Gene Expression in Arabidopsis. Plant Physiol. 2018;178(1):40-53.

9. Samalova M, Kirchhelle C, Moore I. Universal Methods for Transgene Induction Using the Dexamethasone-Inducible Transcription Activation System p0p6/LhGR in Arabidopsis and Other Plant Species. Curr Protoc Plant Biol. 2019;4(2):e20089.

10. Moore I, Samalova M, Kurup S. Transactivated and chemically inducible gene expression in plants. Plant J. 2006;45(4):651-83.

11. Gomez MD, Barro-Trastoy D, Escoms E, Saura-Sanchez M, Sanchez I, Briones-Moreno A, et al. Gibberellins negatively modulate ovule number in plants. Development. 2018;145(13).

12. Kawashima T, Maruyama D, Shagirov M, Li J, Hamamura Y, Yelagandula R, et al. Dynamic F-actin movement is essential for fertilization in Arabidopsis thaliana. Elife. 2014;3.

13. Shi D, Lebovka I, Lopez-Salmeron V, Sanchez P, Greb T. Bifacial cambium stem cells generate xylem and phloem during radial plant growth. Development. 2019;146(1).

14. Caggiano MP, Yu X, Bhatia N, Larsson A, Ram H, Ohno CK, et al. Cell type boundaries organize plant development. Elife. 2017;6. 
15. Kannangara R, Branigan C, Liu Y, Penfield T, Rao V, Mouille G, et al. The transcription factor WIN1/SHN1 regulates Cutin biosynthesis in Arabidopsis thaliana. Plant Cell. 2007;19(4):1278-94.

16. Baroux C, Blanvillain R, Moore IR, Gallois P. Transactivation of BARNASE under the AtLTP1 promoter affects the basal pole of the embryo and shoot development of the adult plant in Arabidopsis. Plant J. 2001;28(5):503-15.

17. Schoof H, Lenhard M, Haecker A, Mayer KF, Jürgens G, Laux T. The stem cell population of Arabidopsis shoot meristems in maintained by a regulatory loop between the CLAVATA and WUSCHEL genes. Cell. 2000;100(6):635-44.

18. Binarova P, Cenklova V, Prochazkova J, Doskocilova A, Volc J, Vrlik M, et al. Gamma-tubulin is essential for acentrosomal microtubule nucleation and coordination of late mitotic events in Arabidopsis. Plant Cell. 2006;18(5):1199-212.

19. Pinto SC, Mendes MA, Coimbra S, Tucker MR. Revisiting the Female Germline and Its Expanding Toolbox. Trends Plant Sci. 2019;24(5):455-67.

20. Schneitz K, Hülskamp M, Pruitt RE. Wild-type ovule development in Arabidopsis thaliana: a light microscope study of cleared whole-mount tissue. Plant J. 1995;7(5):731-49.

21. She W, Grimanelli D, Rutowicz K, Whitehead MW, Puzio M, Kotlinski M, et al. Chromatin reprogramming during the somatic-to-reproductive cell fate transition in plants. Development. 2013;140(19):4008-19.

22. Clough SJ, Bent AF. Floral dip: a simplified method for Agrobacterium-mediated transformation of Arabidopsis thaliana. Plant J. 1998;16(6):735-43.

23. Gross-Hardt R, Lenhard M, Laux T. WUSCHEL signaling functions in interregional communication during Arabidopsis ovule development. Genes Dev. 2002;16(9):1129-38.

24. Tucker MR, Okada T, Hu Y, Scholefield A, Taylor JM, Koltunow AM. Somatic small RNA pathways promote the mitotic events of megagametogenesis during female reproductive development in Arabidopsis. Development. 2012;139(8):1399-404.

25. Payne T, Johnson SD, Koltunow AM. KNUCKLES (KNU) encodes a $\mathrm{C} 2 \mathrm{H} 2$ zinc-finger protein that regulates development of basal pattern elements of the Arabidopsis gynoecium. Development. 2004;131(15):3737-49.

26. Mendocilla Sato E, Baroux C. Analysis of 3D Cellular Organization of Fixed Plant Tissues Using a User-guided Platform for Image Segmentation. Bio-protocol. 2017;7(12):e2355.

27. Tofanelli R, Vijayan A, Scholz S, Schneitz K. Protocol for rapid clearing and staining of fixed Arabidopsis ovules for improved imaging by confocal laser scanning microscopy. Plant Methods. 2019;15:120.

28. Shaner NC, Lin MZ, McKeown MR, Steinbach PA, Hazelwood KL, Davidson MW, et al. Improving the photostability of bright monomeric orange and red fluorescent proteins. Nat Methods. 2008;5(6):545-51.

29. Muller S, Livanos P. Plant Kinesin-12: Localization Heterogeneity and Functional Implications. Int J Mol Sci. 2019;20(17). 
30. Boehm JS, Zhao JJ, Yao J, Kim SY, Firestein R, Dunn IF, et al. Integrative genomic approaches identify IKBKE as a breast cancer oncogene. Cell. 2007;129(6):1065-79.

31. Ito T, Wellmer F, Yu H, Das P, Ito N, Alves-Ferreira M, et al. The homeotic protein AGAMOUS controls microsporogenesis by regulation of SPOROCYTELESS. Nature. 2004;430(6997):356-60.

32. Yang WC, Ye D, Xu J, Sundaresan V. The SPOROCYTELESS gene of Arabidopsis is required for initiation of sporogenesis and encodes a novel nuclear protein. Genes Dev. 1999;13(16):2108-17.

33. Ossowski S, Schwab R, Weigel D. Gene silencing in plants using artificial microRNAs and other small RNAs. Plant J. 2008;53(4):674-90.

34. Musielak TJ, Schenkel L, Kolb M, Henschen A, Bayer M. A simple and versatile cell wall staining protocol to study plant reproduction. Plant Reprod. 2015;28(3-4):161-9.

35. Burton RA, Shirley NJ, King BJ, Harvey AJ, Fincher GB. The CesA gene family of barley. Quantitative analysis of transcripts reveals two groups of co-expressed genes. Plant Physiol. 2004;134(1):22436.

36. Hernandez-Lagana E, Mosca G, Sato EM, Pires N, Frey A, Giraldo-Fonseca A, et al. Organ geometry channels cell fate in the Arabidopsis ovule primordium. bioRxiv. 2020:2020.07.30.226670.

37. Yadegari R, Drews GN. Female gametophyte development. Plant Cell. 2004;16(Suppl):133-41.

38. Smyth DR, Bowman JL, Meyerowitz EM. Early flower development in Arabidopsis. Plant Cell. 1990;2(8):755-67.

\section{Figures}
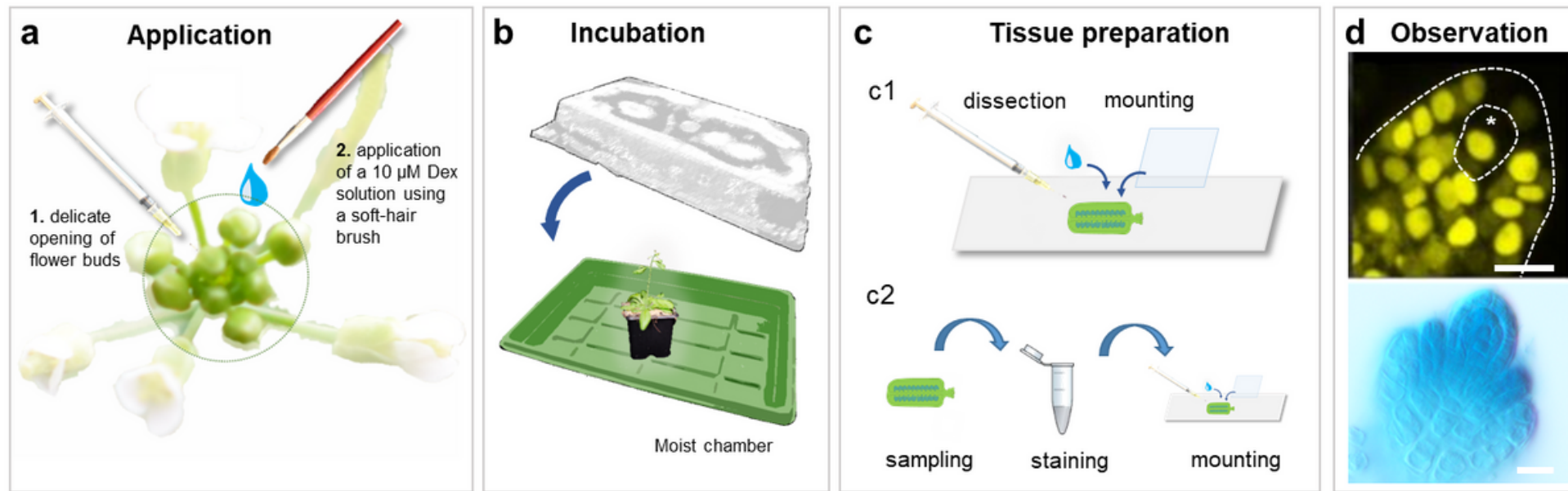

\section{Figure 1}

Schematic representation of steps involved to induce ovules of Arabidopsis thaliana with Dex. (a) Single floral buds are gently opened with dissecting needles under the stereomicroscope to allow for a good exposure of the carpel to the induction solution applied with a paint brush with soft hairs. (b) Plants are kept overnight in a covered tray, laying side-way, to prevent drying. Carpels are sampled 16hrs post 
induction (hpi) either for direct microscopy analysis (c1, mounting on slide) or for histochemical assay for GUS reporter detection (c2). (D) Representative examples of induced reporter genes:

RPS5a >>nls3xmVenus (top) and RPS5a>>GUS (bottom).

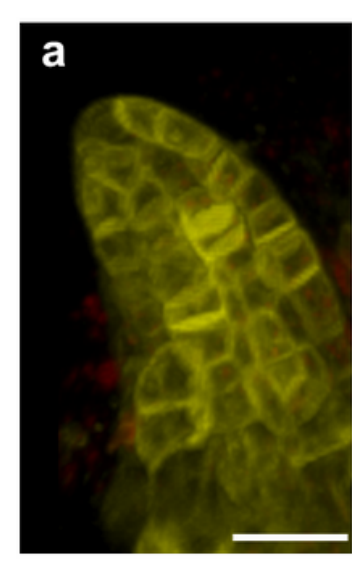

YFP- $\Delta$ Kinesin

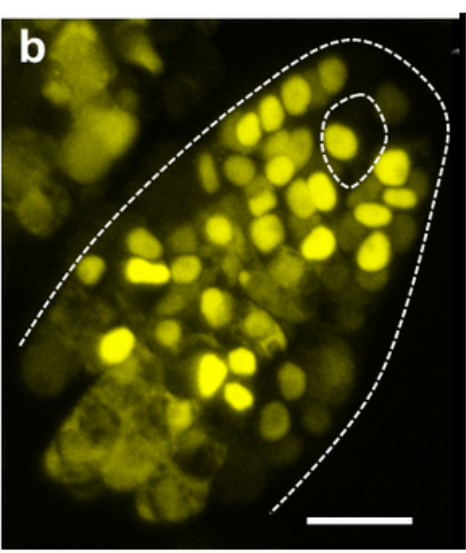

n/s $3 \times$ Venus

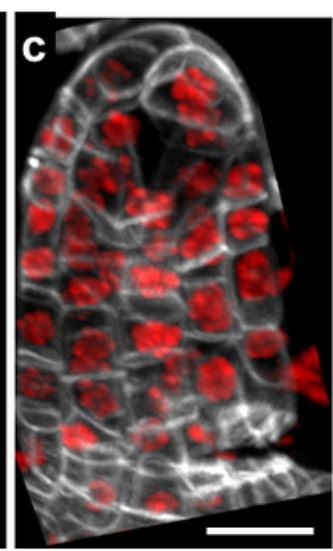

H1.1-RFP

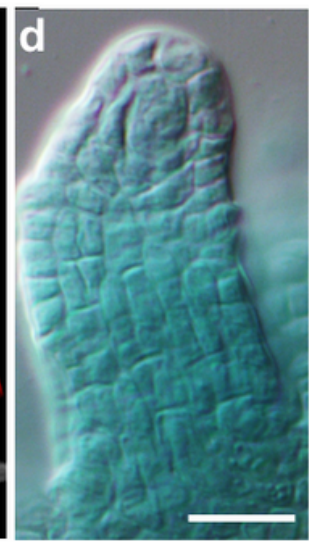

GUS

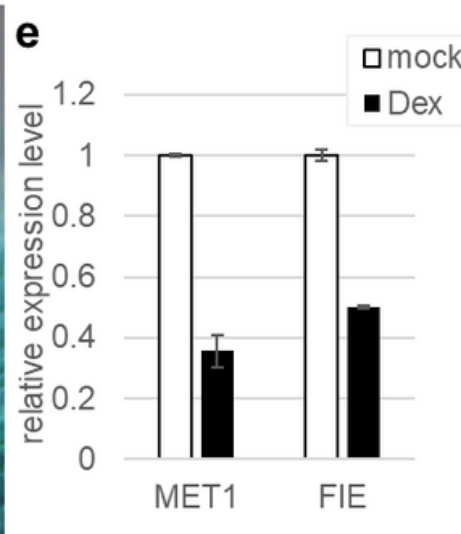

amiRNA

\section{Figure 2}

Induction of a variety of constructs with distinct subcellular targets (a) RPS5a >>YFP- $\triangle$ kinesin, 24 hpi; (b) RPS5a >>nls3xmVenus, 5 dpi. (c) RPS5a>>H1.1*-RFP, 5 dpi. (d) RPS5a>>GUS, 24 hpi, (e) RT-PCR showing FIE and MET1 expression levels in stage 8-10 flower buds (38) expressing the inducible SPL >>amiRFIE or SPL >>amiRMET1, respectively. See Additional File 1 for data source (e) scale bar $=10 \mu \mathrm{m}$.

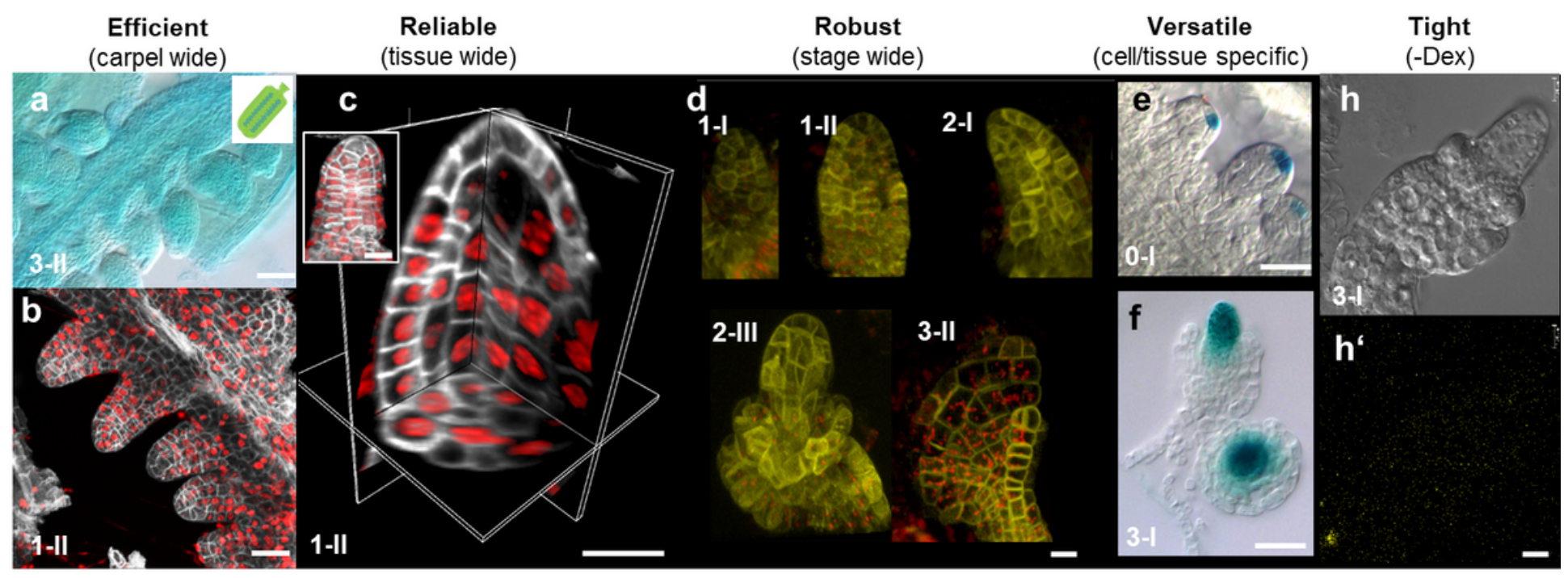

\section{Figure 3}

Induction efficiency in ovules. (a) RPS5a>>GUS, (b,c) RPS5a>>H1.1-RFP (d) RPS5a >>YFP- $\triangle$ kinesin, (e-h) SPL >>GUS, (h-h') RPS5a >>YFP- $\triangle$ kinesin in Mock condition. (a-f) Time post Dex induction: 24h (a), 16h (bh). Confocal images: partial projections (b,d), full projection (c, inset), combined orthogonal sections (c);

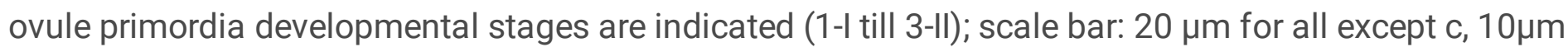




\section{a Developmental progression}

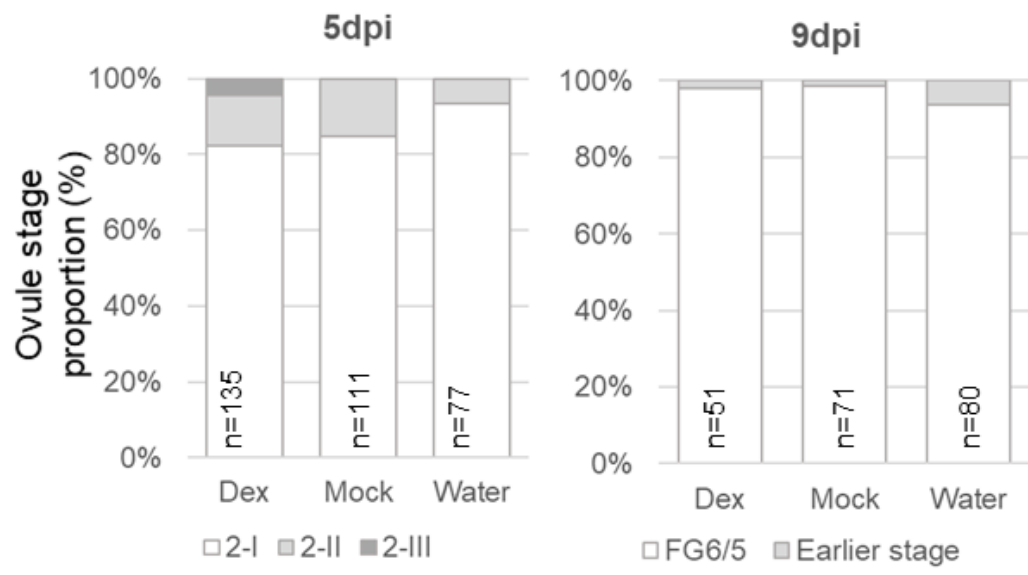

\section{b Functional conditions}

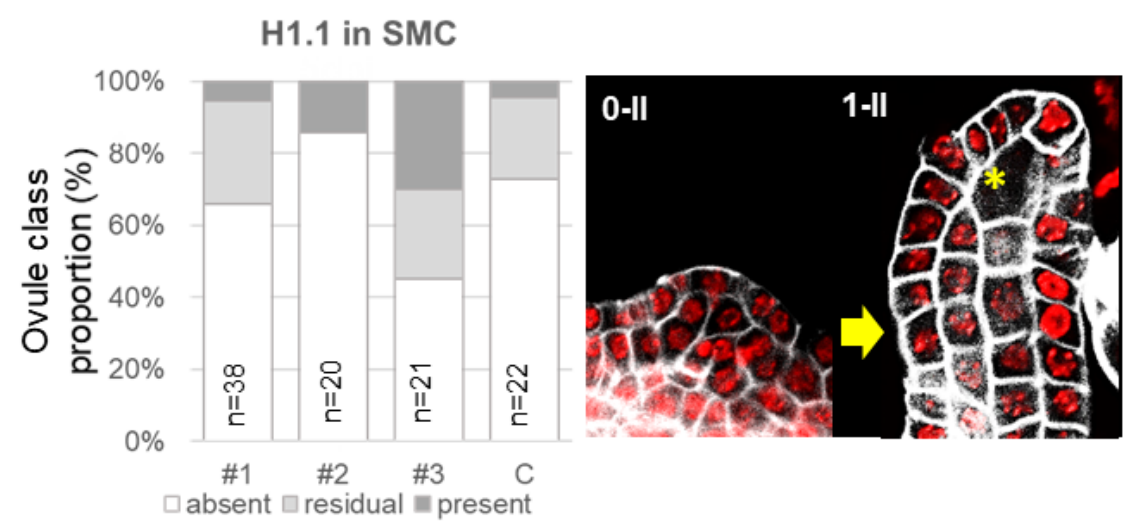

\section{Fertility analysis}

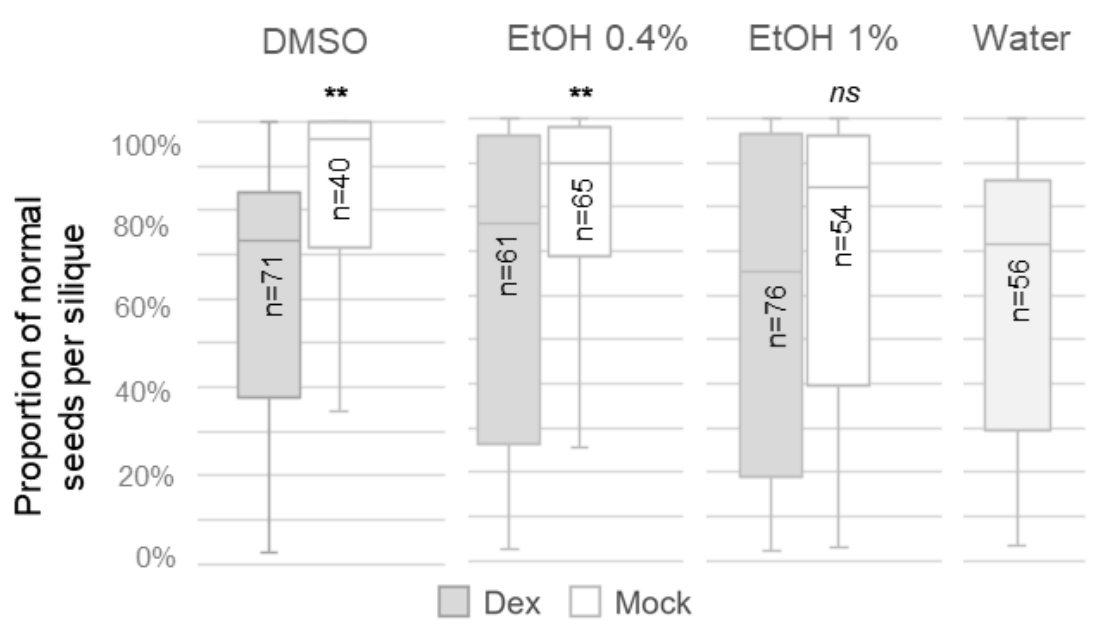

\section{Figure 4}

No or moderate impact of the Dex-treatment on ovule development and fertility The impact of the Dex treatment (including mechanical handling and chemical application) was assessed in three ways. (a) The developmental progression of ovules following treatment was monitored by scoring ovule stages at 5 and $9 \mathrm{dpi}$ (dpi, days post induction) following nomenclature in $(20,37), n=$ number of ovules. (b) The functional impact of s were assessed by monitoring SMC differentiation in the ovule, notably the eviction 
of $\mathrm{H} 1.1$ at stage 1-II as described (21), n=number of stage 1-II ovule primordia, white/grey ovule classes refer to the absence, presence or residual presence of $\mathrm{H} 1.1$ in the SMC following induction of the RPS5a >>H1.1-RFP line (5dpi), \#1-3, three independent lines, C, control (H1.1::H1.1-RFP non inducible line). (c) The impact on seed set (fertility) was monitored by scoring the proportion of normal seeds per silique in treated plants. n, number of siliques. Total number of seeds: $3474,2042,2966,3273,3407,2518,2641$, respectively left to right. See Additional File 1 for source data.

\section{Supplementary Files}

This is a list of supplementary files associated with this preprint. Click to download.

- AdditionalFile1.xlsx 